\title{
Critical adsorption of an end-grafted diblock copolymer on a flat surface
}

\author{
Hong Li ${ }^{1}$, Chang-Ji Qian ${ }^{1}$, Jian-Hua Huang ${ }^{2}$ and Meng-Bo Luo ${ }^{3}$ \\ The critical adsorption of a single flexible diblock copolymer $\mathbf{A}_{n} \mathbf{B}_{m}$ on a flat surface selective to block $\mathrm{A}$ is investigated by using \\ the Monte Carlo simulation method. The surface is modeled as attractive to block $A$ but inert to block $B$, whereas the head \\ monomer $\mathrm{A}$ is grafted on the surface. The results show that the critical adsorption temperature, $T_{\mathrm{c}}$, and the crossover exponent, \\ $\phi$, are the same as that of homogeneous polymer $A_{n}$ for large $n$ values. Although the B block decreases the A block surface- \\ contact number and lowers the adsorption temperature of the finitely long polymer, the effect is eliminated with the increase \\ in the length of the A block. However, we find that the B block has no influence on the mean asphericity parameter of the \\ A block at $T_{\mathrm{c}}$. \\ Polymer Journal (2015) 47, 53-58; doi:10.1038/pj.2014.86; published online 15 October 2014
}

\section{INTRODUCTION}

Polymers tethered to surfaces have an important role in various biological processes. ${ }^{1-4}$ The adsorption of the polymer can modify the surface and interfacial properties, and the degree is dependent on the number of adsorbed monomers. The polymer tethered to the surface can improve or prevent the adsorption of proteins on the surface. For example, the design of biocompatible materials requires reducing the adsorption of proteins on the surface of the material, leading to low platelet adhesion that reduces thrombus formation. ${ }^{2}$ Therefore, understanding the physics of adsorption and desorption of the polymer with one end tethered to a surface is useful to design special surfaces with improved biocompatibility. ${ }^{1}$ Surfaces coated with polymers are also relevant to many applications in industrial and biological technologies such as chromatography, ${ }^{5,6}$ polymer compatibilization, ${ }^{7,8}$ medical implants and bioaffinity sensors. ${ }^{9}$ The behavior of polymers near attractive surfaces has been extensively studied in theory and by computer simulation. ${ }^{10-13}$

A single homogeneous polymer interacting with a flat surface has been well investigated to identify the phase transition from a desorbed state to an adsorbed state with a temperature decrease beyond a critical adsorption point (CAP) $T_{\mathrm{c}} \cdot{ }^{11-19}$ Crossover scaling laws for a variety of quantities below, above and at the CAP have been formulated and verified by Monte Carlo (MC) simulation of self-avoiding walks. ${ }^{11}$ Every monomer contacting the surface is assigned a monomer-surface attraction energy $-E$ (or scaled energy $\varepsilon=E / k_{\mathrm{B}} T$ with $k_{\mathrm{B}}$ being the Boltzmann constant and $T$ the temperature). The CAP can be interpreted as a second-order phase transition at the temperature $T=T_{\mathrm{c}}$ in the thermodynamic limit when the chain length $N$ proceeds to infinity. The number of surface contacts, $M$, scales as $M \sim N^{\phi}$ at the CAP. Much attention has been paid to the location of the CAP, the scaling behavior at the CAP and the determination of the crossover exponent, $\phi$. The crossover exponent, $\phi$, is somewhat disputable and lies in a range between $\phi=0.59^{11,14,15}$ and $\phi=0.5 .^{14,20-22}$ Recently, the scaling relationship for the adsorption of a single chain has been tested by MC simulation on a cubic lattice, ${ }^{15-19}$ and the adsorption transition of a polymer is well known. ${ }^{17-22}$

Although extensive investigations of the CAP of homogeneous polymers have been carried out, there are few studies on the adsorption transition of copolymers. The self-consistent field theory was generalized to describe the adsorption of block copolymers. ${ }^{23}$ The density profile of the adsorbing block $\mathrm{A}$ for the diblock copolymer $\mathrm{A}_{n} \mathrm{~B}_{m}$ is similar to that of the homogeneous polymer $\mathrm{A}_{n}$. Then, the less adsorbing or non-adsorbing block $\mathrm{B}$ exists as a dangling tail. ${ }^{23}$ The scaling properties of copolymers in the vicinity of the CAP have been investigated. ${ }^{24-27}$ For random copolymers, the critical adsorption temperature increases with an increase in the fraction of adsorptive monomers of the chain while the crossover exponent $\phi=0.59$ remains constant. ${ }^{25}$ In recent study, the adsorption of a regular multiblock $A B$ copolymer on a solid planar surface was mapped on an effective homogeneous polymer adsorption problem. ${ }^{24,26}$ The CAP and the fraction of adsorbed monomers were found to be dependent on both the chain length and the block size of the attractive block A. ${ }^{24}$

In the present study, the behavior of an end-grafted diblock copolymer $\mathrm{A}_{n} \mathrm{~B}_{m}$ with the head monomer $\mathrm{A}$ tethered to a flat surface is simulated using the MC method. We consider a specific case where the surface is attractive to monomer A but inert to monomer B, which is a limitation of a general case where the surface attraction of monomer A is stronger than that of monomer B. We have calculated the mean number of monomer $A$ in contact with the surface. The critical behavior of block $A$ is studied using finite-size scaling analysis

${ }^{1}$ Department of Physics, Wenzhou University, Wenzhou, China; ${ }^{2}$ Department of Chemistry, Zhejiang Sci-Tech University, Hangzhou, China and ${ }^{3}$ Department of Physics, Zhejiang University, Hangzhou, China

Correspondence: Professor C-J Qian, Department of Physics, Wenzhou University, Wenzhou, Zhejiang 325035, China.

E-mail: qcj@wzu.edu.cn

Received 29 April 2014; revised 14 August 2014; accepted 15 August 2014; published online 15 October 2014 
on the mean contact number. We found that the critical adsorption temperature of $\mathrm{A}_{n} \mathrm{~B}_{m}$ is the same as that of the homogeneous polymer $\mathrm{A}_{n}$ if $n$ is large. Our results indicate that the tail block $\mathrm{B}$ does not change the CAP of $\mathrm{A}_{n} \mathrm{~B}_{m}$ if the surface attraction of monomer $\mathrm{A}$ is stronger.

\section{MATERIALS AND METHODS}

The diblock copolymer $\mathrm{A}_{n} \mathrm{~B}_{m}$ is embedded in a simple cubic lattice. The chain comprises $n$ type-A monomers and $m$ type-B monomers consecutively linked with fluctuating bonds. The bond length between successive monomers along a chain can be taken from the set $\{(1,0,0),(1,1,0),(1,1,1)\}$ by symmetry operations of the simple cubic lattice. ${ }^{28}$ We adopt a self-avoiding walk chain with each monomer occupying one site of the lattice. The flat surface located at $z=0$ is assumed to be infinitely large and impenetrable to the copolymer chain. Here, the direction $z$ is perpendicular to the flat surface. The copolymer chain is restricted to lie in the upper half space $(z>0)$, and the first monomer $\mathrm{A}$ is fixed at position $(0,0,1)$ that contacts the center of the flat surface $(0,0,0)$. We use a large simulation system along the $x y$ directions parallel to the surface and along the $z$ direction perpendicular to the surface. Periodic boundary conditions are applied in the $x$ and $y$ directions. In addition, the box size along the $x, y$ and $z$ directions is larger than the polymer length $n+m$.

A monomer located at the layer $z=1$ is named the surface-contacted monomer. However, monomers $\mathrm{A}$ and $\mathrm{B}$ contacting the surface have different interaction energies $E_{\mathrm{A}}$ and $E_{\mathrm{B}}$, respectively. In this work, we consider a copolymer with a stronger surface attraction of monomer A. Therefore, we assign an attractive energy $E_{\mathrm{A}}=-E$ for surface-contacted monomers $\mathrm{A}$, whereas $E_{\mathrm{B}}=0$ for monomers $\mathrm{B}$. This parameter set is a limitation for copolymer with stronger surface attraction of monomer $\mathrm{A}$, that is, $\left|E_{\mathrm{A}}\right|>\left|E_{\mathrm{B}}\right|$. The interaction between two non-bonded monomers of the same or the opposite type is purely repulsive and it is assigned as $E_{\mathrm{AA}}=E_{\mathrm{BB}}=E_{\mathrm{AB}}=0$ in the simulation.

At the beginning of the $\mathrm{MC}$ simulation, a self-avoiding walk diblock copolymer chain $\mathrm{A}_{n} \mathrm{~B}_{m}$ is grown using the Rosenbluth-Rosenbluth growing method $^{29}$ with the first monomer A fixed at the surface. After creating the whole copolymer chain, we let it undergo a series of Brownian motion. In the dynamic model, a monomer is chosen randomly and a move is attempted for the monomer moving one lattice unit choosen randomly from one of the six directions $\pm x, \pm y, \pm z$. This trial move will be accepted if the following five conditions are simultaneously satisfied: (1) the new site is located above the surface $(z>0)$, (2) the new site is not occupied by any other monomers, (3) the new bond vectors belong to the allowed bond set, (4) no bonds cross and (5) the Boltzmann factor $\exp \left(-\Delta E / k_{\mathrm{B}} T\right)>p$, where $p$ is a random number uniformly distributed in $(0,1)$ and $\Delta E$ is the change in energy after the trial move. In the simulation, we set the interaction strength $E$ and the Boltzmann constant $k_{\mathrm{B}}$ as unity, that is, $E=1$. Therefore, the unit for temperature is $E / k_{\mathrm{B}}$. In one MC step (MCS), all of the monomers in the polymer chain attempt to move once.

We adopt an annealing method to simulate polymer properties at different temperatures. Starting from a high temperature $T=8$, we slowly decrease the system temperature, $T$. The temperature step $\Delta T$ is chosen as small as 0.05 near $T_{\mathrm{c}}$, whereas a slightly greater value of $\Delta T$ is chosen further from $T_{\mathrm{c}}$. The final configuration at the previous temperature is used as the initial configuration for the subsequent temperature. At each $T$, the copolymer is first equilibrated for $\sim \tau=2.5 N^{2.13}$ MCS. $^{14}$ Then, we record polymer conformations at every $0.1 \tau$ MCS in the next $100 \tau$ MCS. Therefore, we collect 1000 conformations in each run. In our simulation, 2000 independent runs are used, and then the final results are averaged over $2 \times 10^{6}$ independent configuration samples.

\section{RESULTS AND DISCUSSION}

\section{Critical adsorption}

The CAP of polymers can be determined by analyzing the number of surface-contact monomers $M$ at different temperatures. To this end, the surface-contact numbers of the diblock copolymer $\mathrm{A}_{n} \mathrm{~B}_{m}$ are calculated. The surface-contact number of monomer $B$ is close to 0 because of the non-attractive property of $\mathrm{B}$, whereas that of monomer A is relatively large and dependent on the temperature. Therefore, the
A block is strongly adsorbed on the surface at low temperatures, whereas the B block dangles above the surface. In this work, we investigated how the adsorption behavior of the A block is affected by the tail block B.

We at first consider a simple case with $m=n$. The relationship between the mean surface-contact number of monomer $\mathrm{A},\left\langle M_{\mathrm{A}}\right\rangle$, and the length of the block $\mathrm{A}, n$, is studied. For the adsorption of the homogeneous polymer $\mathrm{A}_{n}$, the surface-contact number $M$ can be expressed as a finite-size scaling against the chain length $n$ and the temperature, $T .{ }^{17,30}$ We apply finite-size scaling analysis for the adsorption of the $\mathrm{A}$ block in the diblock copolymer $\mathrm{A}_{n} \mathrm{~B}_{n}$. Thus, $<M_{\mathrm{A}}>$ can be expressed as

$$
\begin{aligned}
<M_{\mathrm{A}}>(T, n)= & n^{\phi}\left(a_{0}+a_{1}\left(T-T_{\mathrm{c}}\right) n^{1 / \delta}\right. \\
& \left.+O\left(\left(T-T_{\mathrm{c}}\right) n^{1 / \delta}\right)^{2}\right),
\end{aligned}
$$

where $\phi$ is the crossover exponent and $T_{\mathrm{c}}$ is the critical adsorption temperature. $\left\langle M_{\mathrm{A}}\right\rangle$ shows different behaviors at temperatures above and below the critical point $T_{\mathrm{c}}$ because the second term $\left(T-T_{\mathrm{c}}\right)(n+1)^{1 / \delta}$ in the scaling form (1) changes sign for temperature, $T$, of annealing from $T>T_{\mathrm{c}}$ to $T<T_{\mathrm{c}}$. Therefore, $\left\langle M_{\mathrm{A}}>\right.$ has the best power law behavior at $T=T_{\mathrm{c}}$, which can be expressed as:

$$
<M_{\mathrm{A}}>=a_{0} n^{\phi} .
$$

Figure 1 presents the dependence of $\left\langle M_{\mathrm{A}}\right\rangle$ on $n$ at different temperatures for the diblock copolymer $\mathrm{A}_{n} \mathrm{~B}_{n}$. One can see that the upward concave curves at low temperatures $(T=1.45,1.60)$ change to downward convex curves at high temperatures $(T=1.65,1.80)$. Therefore, the critical adsorption temperature, $T_{\mathcal{c}}$, of the A block is located among the region $(1.60,1.65)$ according to the finite-size scaling theory. ${ }^{17,18,30}$ The location of $T_{\mathrm{c}}$ is estimated by analyzing $\left\langle M_{\mathrm{A}}\right\rangle$ using the finite-size scaling theory. The values of $\left\langle M_{\mathrm{A}}\right\rangle$ at other temperatures in the interval $(1.60,1.65)$ can be obtained by quadratic interpolation from the simulation data. The best power law fit is achieved at $T=1.625$ for the A block of the diblock copolymer $\mathrm{A}_{n} \mathrm{~B}_{n}$, indicating that $T_{\mathrm{c}}=1.625$ for the infinitely long copolymer $\mathrm{A}_{n} \mathrm{~B}_{n}$. It is interesting that the $T_{\mathrm{c}}$ of the long $\mathrm{A}_{n} \mathrm{~B}_{n}$ is the same as that of a homogeneous polymer with the same model. ${ }^{17}$ At $T_{\mathrm{c}}=1.625$, we obtain the exponent $\phi=0.51$, which is also close to that of the homogeneous polymer $\phi=0.52 .{ }^{17}$ The results clearly show that the tail block $\mathrm{B}$ does not change the CAP and critical exponents of the infinitely long copolymer $\mathrm{A}_{n} \mathrm{~B}_{n}$ if the surface attraction of monomer $\mathrm{A}$ is stronger.

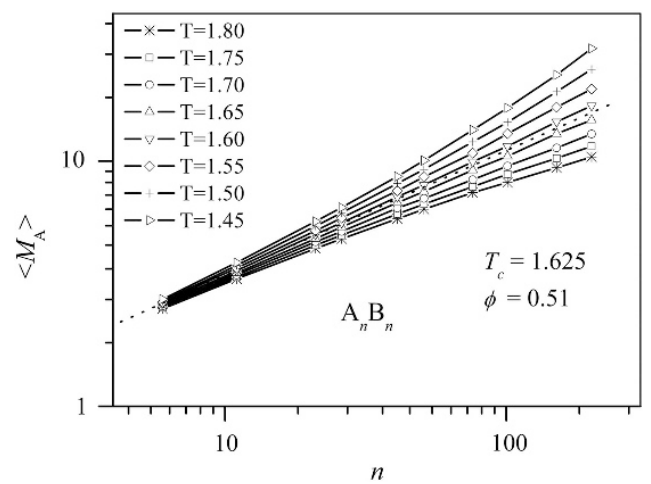

Figure 1 Log-log plot of the mean number of surface contacts $\left\langle M_{\mathrm{A}}\right\rangle$ versus the block length $n$ at temperatures $T$ from 1.45 to 1.80 . Here, $n$ is varied from 5 to 200 . The straight, dotted line at $T_{\mathrm{C}}=1.625$ is a linear fit. 
However, the tail block B can change the contact number as well as conformational properties of the finitely long copolymer $\mathrm{A}_{n} \mathrm{~B}_{n}$. We find that the $\left\langle M_{\mathrm{A}}>\right.$ of $\mathrm{A}_{n} \mathrm{~B}_{n}$ is smaller than that of $\mathrm{A}_{n}$ because of the tail block $B$. Figure 2 presents the contact number fraction of monomer $\mathrm{A}<M_{\mathrm{A}}>/ n$ as a function of the temperature, $T$, for the different block lengths $n=25,50,100$ and 200. The $\left\langle M_{\mathrm{A}}\right\rangle / n$ clearly increases below $T_{\mathrm{c}}$. We find that the $\left\langle M_{\mathrm{A}}\right\rangle / n$ decreases with an increase in $n$ at high temperatures, whereas it is roughly independent of $n$ at low temperatures. These results indicate a second-order adsorption transition for the long copolymer $\mathrm{A}_{n} \mathrm{~B}_{n}$, in agreement with that of the homogeneous polymer. ${ }^{11,17}$ To investigate the effect of the block $\mathrm{B}$, we calculated the value of $f_{<M>}$ for the contact number of $\mathrm{A}_{n} \mathrm{~B}_{n}$ compared with that of $\mathrm{A}_{n}$ :

$$
f_{<M>}=\frac{<M_{\mathrm{A}}>{ }_{\mathrm{A}_{n} \mathrm{~B}_{n}}}{<M_{\mathrm{A}}>_{\mathrm{A}_{n}}}
$$

This value is a ratio of the contact number of monomer $\mathrm{A}$ in the diblock copolymer $\mathrm{A}_{n} \mathrm{~B}_{n}$ to that of the homogeneous polymer $\mathrm{A}_{n}$. The relationships between the ratio $f_{\langle M\rangle}$ and the temperature, $T$, are shown in the inset of Figure 2. One can see that $f_{<M\rangle}<1$, indicating that the non-adsorbed B block weakens the adsorption of the A block. Interestingly, the ratio $f_{<M>}$ exists at a minimum at $T=T_{\mathrm{c}}$ and is roughly independent of the block length $n$ at $T=T_{\mathrm{c}}$. Specifically, we have $\left.<M_{\mathrm{A}}>_{\mathrm{A}_{n} \mathrm{~B}_{n}} \propto<M_{\mathrm{A}}\right\rangle_{\mathrm{A}_{n}}$ at $T=T_{\mathrm{c}}$. This result can explain why $T_{\mathcal{c}}$ and $\phi$ are the same for the copolymer $\mathrm{A}_{n} \mathrm{~B}_{n}$ and for the homogeneous polymer $\mathrm{A}_{n}$. However, the ratio $f_{<M>}$ varies with the A block length $n$ at other temperatures deviating from $T_{\mathrm{c}}$. The ratio $f_{<M>}$ increases with an increase in the length $n$, meaning that the adsorption properties of the A block are less affected by the B block for the long-chain copolymer.

Next, we investigate a general case for the diblock copolymer $A_{n} B_{m}$ to study the influence of the B block on the adsorption of the A block. We compared the dependence of the surface-contact number of the A block, $\left\langle M_{\mathrm{A}}\right\rangle$, on the length of the A block, $n$, for three chains $\mathrm{A}_{n}$, $\mathrm{A}_{n} \mathrm{~B}_{10}$ and $\mathrm{A}_{n} \mathrm{~B}_{20}$ at the critical adsorption temperature $T_{\mathrm{c}}=1.625$. The double logarithmic relationship for $\left\langle M_{\mathrm{A}}\right\rangle-n$ is shown in Figure 3. We find that $\left\langle M_{\mathrm{A}}\right\rangle$ decreases with an increase in the length of the $\mathrm{B}$ block because the dangling $\mathrm{B}$ block prevents adsorption of $\mathrm{A}$ monomers near the $\mathrm{B}$ block. However, we find that the curves $<M_{\mathrm{A}}>-n$ of $\mathrm{A}_{n} \mathrm{~B}_{10}$ and $\mathrm{A}_{n} \mathrm{~B}_{20}$ become closer to that of $\mathrm{A}_{n}$ with an increase in the block length $n$, meaning that the influence of the $\mathrm{B}$

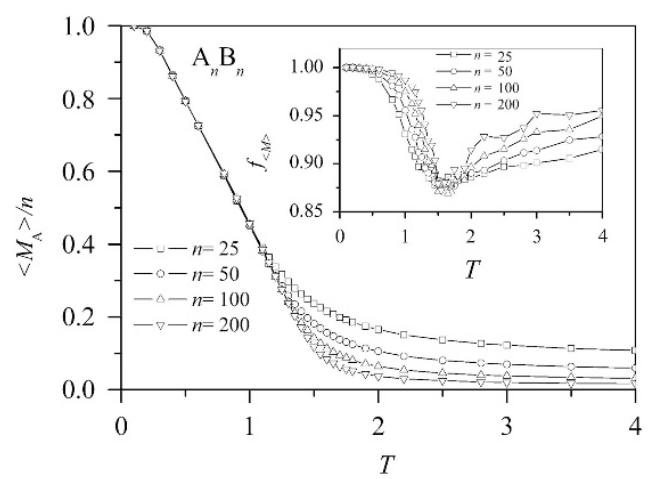

Figure 2 Plot of the mean surface-contact number of monomer $\mathrm{A}\left\langle M_{\mathrm{A}}>\right.$ at different temperatures, $T$, for the diblock copolymer $A_{n} B_{n}$ with different block lengths $n=25,50,100$ and 200. The inset shows the ratio $f_{<M>}$ of the $\left\langle M_{\mathrm{A}}\right\rangle$ of the diblock copolymer $\mathrm{A}_{n} \mathrm{~B}_{n}$ to that of the homogeneous polymer $A_{n}$ at different temperatures. block is eliminated with the increase in $n$. We also find that these curves are roughly parallel at large $n$ values, which is consistent with the same value of $\phi$ for $A_{n}$ and $A_{n} B_{n}$.

In addition, the reduction of the contact number $\left.\Delta<M_{\mathrm{A}}\right\rangle=$ $<M_{\mathrm{A}}>_{\mathrm{A}_{n}}-<M_{\mathrm{A}}>_{\mathrm{A}_{n} \mathrm{~B}_{m}}$ because of the $\mathrm{B}$ block is calculated. The results are presented in the inset of Figure 3 for $\mathrm{A}_{n} \mathrm{~B}_{10}$ and $\mathrm{A}_{n} \mathrm{~B}_{20}$. The $\Delta<M_{\mathrm{A}}>$ increases with $n$ for short chains; however, it tends to be saturated at large $n$ values. This result clearly shows that the influence of the B block is finite and tends to be 0 at large $n$ values. Therefore, one could find that the $\left\langle M_{\mathrm{A}}\right\rangle-n$ curves are roughly parallel at large $n$ values in Figure 3. In addition, one could expect that these curves would overlap at much larger $n$ values. Therefore, we can conclude that the CAP and critical exponents of the diblock copolymer $\mathrm{A}_{n} \mathrm{~B}_{m}$ is the same as that of $\mathrm{A}_{n}$ at large $n$ values.

At finite temperatures, the adsorption of monomer $\mathrm{A}$ is a dynamic process. The influence of the tail block B on the dynamic behavior of the A block is also investigated. The probability that each monomer A is in contact with the surface $P_{\mathrm{A}}$ is calculated for the homogeneous polymer $\mathrm{A}_{100}$ and for the diblock copolymers $\mathrm{A}_{100} \mathrm{~B}_{m}$ with $m=50$ and 200 at $T=1.6$ near CAP. The $P_{\mathrm{A}}$ is calculated as an average contacting event averaged over times and samples. The values of $P_{\mathrm{A}}$ are shown in Figure 4 in a $\log -\log$ plot against the fraction of monomer positioned in the A block. Here, $x=i / n$ represents the fractional position of the $i$ th monomer, where $i$ is a sequence order from the head monomer A $(i=0)$ to the end monomer $\mathrm{A}(i=n-1), P_{\mathrm{A}}(i=0)=1$ is not included in the plot. One can see that $P_{\mathrm{A}}$ decreases with $x$, which can be attributed to two factors: a monomer locates away from the surface as $x$ increases and the rear block behind the monomer prevents its adsorption. The increase in $P_{\mathrm{A}}$ at tail positions for the homogeneous polymer is because of the decrease in length of the rear block. For the homopolymer $\mathrm{A}_{100}$, we find a power-law dependence for $P_{\mathrm{A}}$, that is, $P_{\mathrm{A}} \propto x^{-0.45}$ at $T=1.6$. The power-law behavior changes from a downward curve above $T_{\mathrm{c}}$ to an upward curve below $T_{\mathrm{c}}$. The probability $P_{\mathrm{A}}$ of monomer A close to the B block is strongly affected by the length of the block $\mathrm{B}$. The $P_{\mathrm{A}}$ of monomer A close to the end of the B block clearly decreases because of the non-adsorptive property of the B block. However, the existence of the B block does not affect the adsorption of monomer A close to the grafted monomer $(x=0)$. The length of the rear block A affected by the B block is dependent on the

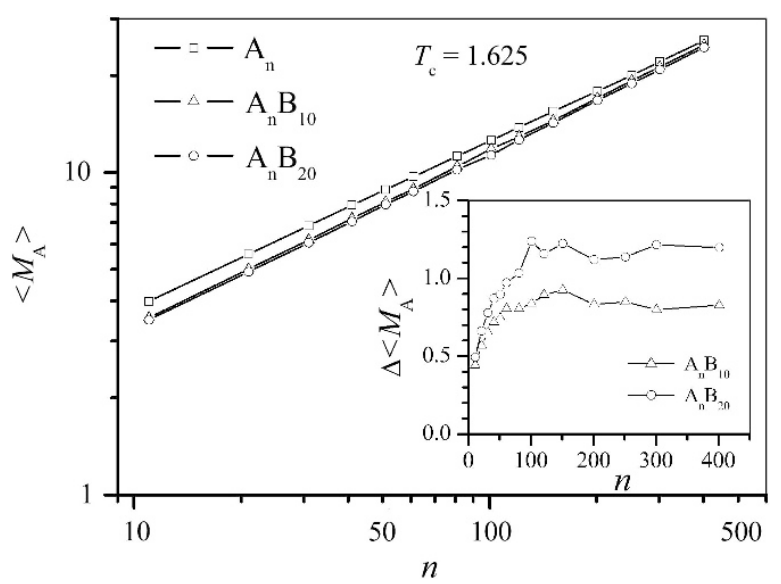

Figure 3 The mean surface-contact number of block $A,\left\langle M_{A}\right\rangle$, for polymer $\mathrm{A}_{n} \mathrm{~B}_{m}$ with $m=0,10$ and 20 at $T_{\mathrm{c}}=1.625$. The inset presents the reduction of contact number $\Delta<M_{\mathrm{A}}>$ because of the $\mathrm{B}$ block. 
length of the B block. The probability $P_{\mathrm{A}}$ of the rear block A decreases with an increase in $m$ for the diblock copolymer $\mathrm{A}_{100} \mathrm{~B}_{m}$.

The influence of the block length of the tail block $\mathrm{B}$ on the adsorption of copolymer $\mathrm{A}_{n} \mathrm{~B}_{m}$ is also studied. The mean surfacecontact numbers $\left\langle M_{\mathrm{A}}\right\rangle$ of the diblock copolymer $\mathrm{A}_{100} \mathrm{~B}_{m}$ are calculated for different values $m$ and at different temperatures. We find that $\left\langle M_{\mathrm{A}}\right\rangle$ is related not only to the temperature, $T$, but also to the length of the $\mathrm{B}$ block. As in the case of $\mathrm{A}_{n} \mathrm{~B}_{n}$, the ratio $f_{<M>}=\frac{\left\langle M_{\mathrm{A}}\right\rangle_{A_{n} \mathrm{~B}_{m}}}{\left\langle M_{\mathrm{A}}\right\rangle_{\mathrm{A}_{n}}}$, which describes the contact number reduction because of the $\mathrm{B}$ block, is also calculated. Figure 5 shows the dependence of $f_{<M>}$ on the temperature, $T$, for the copolymer $\mathrm{A}_{100} \mathrm{~B}_{m}$ with different $m$ values. At a very low temperature, the ratio $f_{<M>}$ is almost constant with respect to $m$. As the temperature, $T$, increases, the ratio $f_{<M>}$ rapidly decreases with an increase in $m$. There is a minimum of the ratio $f_{<M>}$ just at the critical adsorption temperature, $T_{\mathrm{c}}$. These results indicate that the tail block B influences the surface-contact number of monomer A for the diblock copolymer $\mathrm{A}_{n} \mathrm{~B}_{m}$; however, it has no influence on the critical adsorption temperature, $T_{\mathrm{c}}$.

The copolymer $\mathrm{A}_{n} \mathrm{~B}_{m}$ can be separated into two types by comparison of their surface attraction. If the attraction strength of monomer

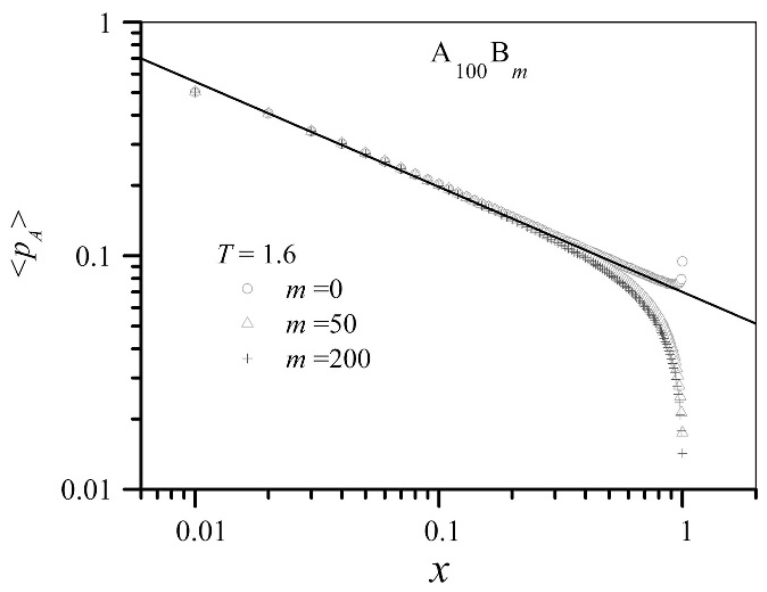

Figure 4 The probability that each monomer $A$ is in contact with the surface for the polymer $A_{n} B_{m}$ with $m=0,50$ and 200 near the critical adsorption temperature $T=1.6$. The slope of the solid line is -0.45 . A full color version of this figure is available at Polymer Journal online.

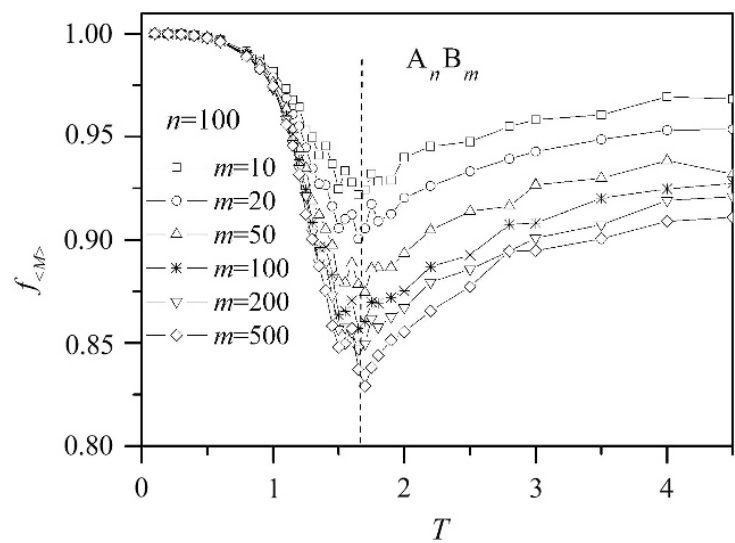

Figure 5 Plots of the ratio $f_{<M}$ of the $\left\langle M_{\mathrm{A}}>\right.$ of diblock copolymer $\mathrm{A}_{100} \mathrm{~B}_{m}$ to that of the homogeneous polymer $A_{100}$ at different temperatures, $T$, for different lengths of the B block, $m$.
$\mathrm{A}$ is stronger than that of monomer $\mathrm{B}$, we can set $E_{\mathrm{AS}}=-1$ and $E_{\mathrm{BS}}=0$ as the limitation, otherwise we set $E_{\mathrm{AS}}=0$ and $E_{\mathrm{BS}}=-1$. For the former case, our simulations show that the $T_{\mathrm{c}}$ of $E_{\mathrm{BS}}=0$ is equal to that of $E_{\mathrm{BS}}=-1$ corresponding to the homogenous polymer $\mathrm{A}_{n}$. Therefore, we conclude that $\mathrm{B}$ block has no effect on the critical adsorption temperature, $T_{\mathrm{c}}$, if $E_{\mathrm{BS}}>-1$. Although we only study the case $E_{\mathrm{AS}}=-1$ and $E_{\mathrm{BS}}=0$, it in fact includes all $E_{\mathrm{BS}}>-1$. For the other case with $E_{\mathrm{AS}}=0$ and $E_{\mathrm{BS}}=-1$ (that is, the limitation for the attraction of $B$ is stronger than $A$ ), the situation differs because the adsorption of $\mathrm{B}$ takes place before that of $\mathrm{A}$. This case is more complicated but deserves further study.

\section{Conformational properties}

We also studied the influence of the non-adsorbed B block on the conformation properties of the A block for the diblock copolymer chains $\mathrm{A}_{n} \mathrm{~B}_{m}$. The dependence of the mean square end-to-end distance of block $\mathrm{A}<R_{\mathrm{A}}^{2}>$ on the temperature, $T$, is shown in Figure 6 for $n=100$ and $m=10,50,100$ and 500. The $\left\langle R_{\mathrm{A}}^{2}\right\rangle$ displays a minimum value near the CAP $T_{c}=1.625$, which is in agreement with the behavior of the homogeneous polymer. ${ }^{17,19}$ Therefore, the place where $\left\langle R_{\mathrm{A}}^{2}\right\rangle$ is minimum is defined as the critical adsorption temperature for a finitely long polymer. ${ }^{19}$ At high temperatures, $T>T_{\mathrm{c}}$, the $\left\langle R_{\mathrm{A}}^{2}>\right.$ changes little with the temperature but increases with an increase in the length of block $B$. At low temperatures, $T<T_{\mathrm{c}}$, the $\left\langle R_{\mathrm{A}}{ }^{2}\right\rangle$ is mainly determined by the temperature. We find that the critical temperature $T_{c}\left(A_{n} B_{m}\right)$ for a finite long chain $A_{n} B_{m}$, where $\left.<R_{\mathrm{A}}{ }^{2}\right\rangle$ is a minimum, is dependent on the length of the A block, $n$ and the length of the B block, $m$. The critical temperature $T_{c}\left(\mathrm{~A}_{n} \mathrm{~B}_{m}\right)$ first decreases with an increase in $m$ for a short B block. When $m$ increases to a certain value $(m=n)$, then the critical temperature $T_{\mathrm{C}}$ $\left(\mathrm{A}_{n} \mathrm{~B}_{m}\right)$ is roughly independent of $m$, as shown in the inset of Figure 6. These results clearly show that the B block influences the critical adsorption temperature of the A block of the diblock copolymer $\mathrm{A}_{n} \mathrm{~B}_{m}$. In other words, the $T_{\mathrm{c}}\left(\mathrm{A}_{n} \mathrm{~B}_{m}\right)$ of the finite copolymer $\mathrm{A}_{n} \mathrm{~B}_{m}$ is dependent on the length of the $\mathrm{B}$ block, although block $\mathrm{B}$ is inerted to the surface.

It is known that the $T_{c}\left(\mathrm{~A}_{n}\right)$ of finite long chain $\mathrm{A}_{n}$ is dependent on the length of the polymer. ${ }^{17-19}$ The dependence of $T_{c}\left(A_{n} B_{m}\right)$ on the length of the $A$ block is checked for copolymer $A_{n} B_{m}$. To this end, we choose $\mathrm{A}_{n} \mathrm{~B}_{n}$ as an example, and $T_{c}\left(\mathrm{~A}_{n} \mathrm{~B}_{n}\right)$ is estimated from the minimum of $\left\langle R_{\mathrm{A}}^{2}\right\rangle$. The dependence of $\left\langle R_{\mathrm{A}}^{2}\right\rangle$ on the temperature, $T$, is plotted in Figure 7 for both $\mathrm{A}_{n} \mathrm{~B}_{n}$ and $\mathrm{A}_{n}$ with $n=100$. Each

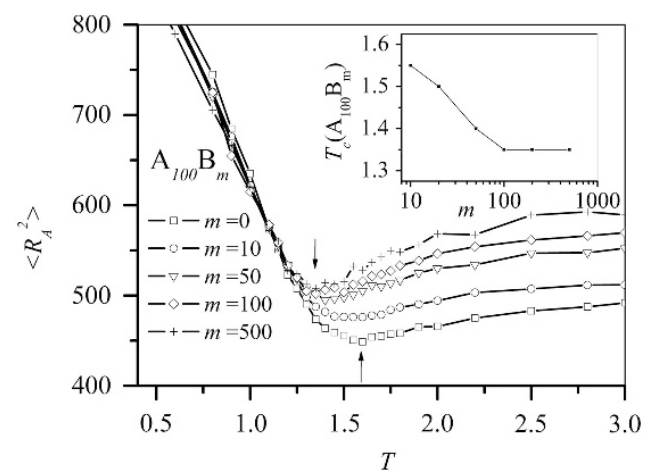

Figure 6 Plots of the mean square end-to-end distance $\left\langle R_{\mathrm{A}}{ }^{2}\right\rangle$ of the $\mathrm{A}$ block versus the temperature, $T$, for the polymer $A_{100} B_{m}$ with different lengths of the $B$ block $m$. The inset shows the effect of the $T_{c}\left(A_{100} B_{m}\right)$ of the diblock copolymer $A_{100} B_{m}$ on the length of block $B$ m. 


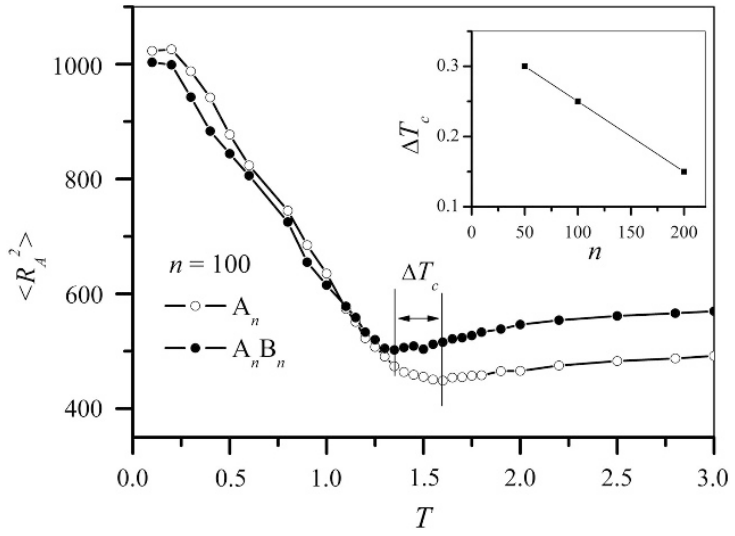

Figure 7 Plots of the mean square end-to-end distance $\left\langle R_{\mathrm{A}}{ }^{2}\right\rangle$ versus temperature, $T$, for the homogeneous polymer $A_{n}$ and for the $A$ block of the diblock copolymer $A_{n} B_{n}$ with $n=100$. The inset presents the critical temperature difference $\Delta T_{c}, \Delta T_{c}=T_{c}\left(A_{n}\right)-T_{c}\left(A_{n} B_{n}\right)$ because of the $B$ block.

curve has a minimum, and the temperature of the minimum is named as the critical adsorption temperature $T_{\mathrm{c}}\left(\mathrm{A}_{n} \mathrm{~B}_{n}\right)$. Again, $T_{\mathrm{c}}\left(\mathrm{A}_{n} \mathrm{~B}_{n}\right)$ is lower than that of $T_{\mathrm{c}}\left(\mathrm{A}_{n}\right)$ because of the influence of the B block. We have calculated the difference $\Delta T_{\mathrm{c}}$ between the two critical adsorption temperatures, which is defined as:

$$
\Delta T_{\mathrm{c}}=T_{\mathrm{c}}\left(\mathrm{A}_{n}\right)-T_{c}\left(\mathrm{~A}_{n} \mathrm{~B}_{n}\right) \text {. }
$$

We find that both $T_{\mathrm{c}}\left(\mathrm{A}_{n}\right)$ and $T_{\mathrm{c}}\left(\mathrm{A}_{n} \mathrm{~B}_{n}\right)$ increase with the length of the A block. However, the difference $\Delta T_{\mathrm{c}}$ decreases with an increase in $n$, as shown in the inset of Figure 7. Our results indicate that the tail block $\mathrm{B}$ influences the location of the minimum of $\left\langle R_{\mathrm{A}}^{2}\right\rangle$ for the $\mathrm{A}$ block of $A_{n} B_{n}$ for a finitely long polymer but has almost no influence on the location of the minimum of $\left\langle R_{\mathrm{A}}{ }^{2}\right\rangle$ for an infinitely long polymer. Therefore, the influence of the $\mathrm{B}$ block is eliminated with the increase in the length of the A block, and $\Delta T_{\mathrm{c}}$ tends to 0 for an infinitely long polymer. This result also explains that the CAP of the diblock copolymer $\mathrm{A}_{n} \mathrm{~B}_{n}$ is the same as that of homogeneous polymer $\mathrm{A}_{n}$ for an infinitely large $n$.

\section{Asphericity parameter}

In addition to the statistical size of the copolymer, the statistical instantaneous shape behavior of the copolymer $\mathrm{A}_{n} \mathrm{~B}_{m}$ near the critical adsorption temperature is investigated. The instantaneous polymer shape can be expressed by the mean asphericity parameter $\langle A\rangle$, which is defined as

$$
<\mathrm{A}>=<\sum_{i>j}^{3}\left(L_{i}^{2}-L_{j}^{2}\right)^{2} / 2\left(\sum_{i=1}^{3} L_{i}^{2}\right)^{2}>
$$

in a three-dimensional (3D) space. ${ }^{17,31,32}$ Here, $L_{1}^{2}, L_{2}^{2}$ and $L_{3}^{2}$ $\left(L_{1}^{2} \leqslant L_{2}^{2} \leqslant L_{3}^{2}\right)$ are the three eigenvalues of the radius of gyration tensor $\mathrm{S}$, which is defined as

$$
S=\frac{1}{n} \sum_{i=1}^{n} s_{i} s_{i}^{T}=\left(\begin{array}{lll}
S_{x x} & S_{x y} & S_{x z} \\
S_{x y} & S_{y y} & S_{y z} \\
S_{x z} & S_{y z} & S_{z z}
\end{array}\right),
$$

where the matrix $s_{i}=\operatorname{col}\left(x_{i}, y_{i}, z_{i}\right)$ represents the position of monomer $i$ in a frame of reference with its origin at the center of mass. ${ }^{31,32} s_{i}{ }^{T}$ is the transposed matrix of $s_{i}$. By this definition, we have $S_{\alpha \beta}=$ $\frac{1}{n} \sum_{i=1}^{n} \alpha_{i} \beta_{i}(\alpha, \beta=x, y, z)$. Specifically, $S_{x x}, S_{y y}$ and $S_{z z}$ are the three components of the mean-square radius of gyration along the $x, y$ and $z$

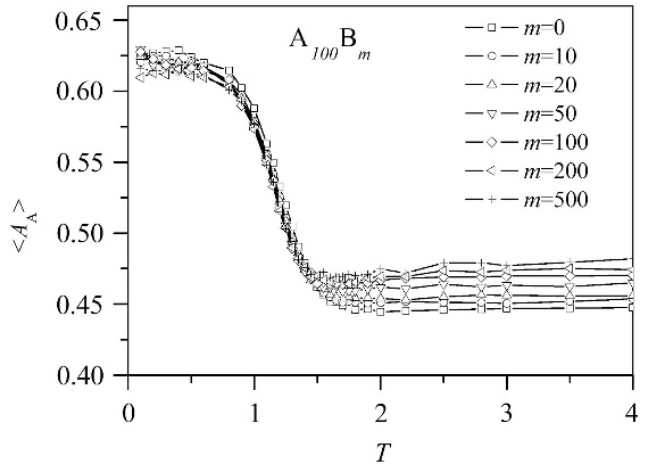

Figure 8 Plot of the mean asphericity parameter $<A>$ of the $A$ block versus the temperature, $T$, for $A_{100} B_{m}$ with different $B$ block lengths, $m$.

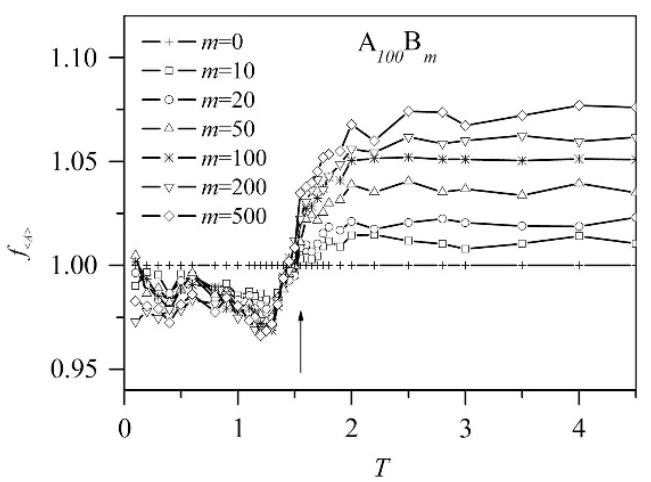

Figure 9 Plot of the ratio $f_{<A}$ between $<A>$ of the diblock copolymer $A_{n} B_{n}$ and that of the homogeneous polymer $A_{n}$ at the temperature, $T$, for different $B$ block lengths, $m$.

directions, respectively. The mean asphericity parameter $\langle\mathrm{A}\rangle$ of the A block in $A_{n} B_{m}$ is calculated at different temperatures. The relationship between $<\mathrm{A}>$ and the temperature, $T$, is presented in Figure 8 for $\mathrm{A}_{n} \mathrm{~B}_{m}$ with $n=100$. $<\mathrm{A}>$ does not change with $T$ at high temperature $T>T_{\mathcal{c}}$; however, it clearly increases below $T_{\mathcal{c}}$ and saturates at low temperatures. The values of $\langle\mathrm{A}\rangle$ change from $\sim 0.45$ at high temperatures to 0.62 at low temperatures, indicating a transition from a $3 \mathrm{D}$ configuration to a two-dimensional (2D) configuration. At high temperatures, $T>T_{\mathcal{C}},<\mathrm{A}>$ increases with an increase in the length of the B block $m$, whereas at low temperature $T<T_{\mathfrak{c}},<\mathrm{A}>$ is roughly independent of $m$. We have checked the influence of the B block on the instantaneous shape of the copolymer by defining a ratio $f_{<\mathrm{A}>}$ as

$$
f_{<A>}=\frac{<\mathrm{A}>_{\mathrm{A}_{n} \mathrm{~B}_{m}}}{<\mathrm{A}>_{\mathrm{A}_{n}}},
$$

where $<\mathrm{A}>{ }_{\mathrm{A}_{n} \mathrm{~B}_{m}}$ is the asphericity parameter of the A block of $\mathrm{A}_{n} \mathrm{~B}_{m}$ and $\langle\mathrm{A}\rangle_{\mathrm{A}_{n}}$ is that of $\mathrm{A}_{n}$, respectively. The dependence of $f_{<\mathrm{A}}>$ on the temperature $T$ is shown in Figure 9. At $T=T_{\mathrm{c}}$, the ratio $f_{<\mathrm{A}>}$ is equal to 1 , indicating that the length of the B block $m$ has no influence on $<\mathrm{A}\rangle_{\mathrm{A}_{n} \mathrm{~B}_{m}}$ at $T_{\mathrm{c}}$. This result is consistent with the asphericity parameter of the homogeneous polymer chain that is independent of chain length at CAP. ${ }^{17}$ At high temperatures, the ratio $g_{<\mathrm{A}>}$ is greater than 1 . The movement of the tail block B drags the A block away from the surface and increases the size of the block A (Figure 7) as well as the asphericity parameter. At low temperatures, the ratio $f_{<\mathrm{A}\rangle}$ is slightly less than 1 . The movement of the tail block $\mathrm{B}$ again drags the $\mathrm{A}$ 
block away from the surface, indicated by the decrease in the adsorption probability with $m$ as shown in Figure 4. This prevents the adsorption of tail monomers in the A block; thus, the size (Figure 7) and the asphericity parameter (Figure 9) decrease with the length of the B block. It is known that a $2 \mathrm{D}$ configuration has a larger asphericity parameter.

We have also investigated the adsorption of the copolymer $\mathrm{A}_{n} \mathrm{~B}_{m}$ with a stronger surface attraction of monomer $B$. In addition, we simulated the copolymer for a limitation case with $E_{\mathrm{A}}=0$ and $E_{\mathrm{B}}=-1$. Interestingly, we find that the critical adsorption temperature is the same as that of the homogeneous polymer when the length of the tail block $m$ tends to be infinity. However, the dependence of the conformational properties on the non-adsorbed block is more complicated. Nevertheless, all of our results show that the critical adsorption of the diblock copolymer $\mathrm{A}_{n} \mathrm{~B}_{m}$ is determined by the block with stronger attraction.

\section{CONCLUSIONS}

Dynamic MC simulations are carried out for investigating the adsorption of a single flexible diblock copolymer chain $\mathrm{A}_{n} \mathrm{~B}_{m}$ with the head monomer A grafted to a flat surface. The surface is impenetrable by the copolymer through the adoption of a selfavoiding walk. This article considers a specific case with attractive surface interactions of monomer $\mathrm{A}$ and non-attractive monomer surface interactions of monomer B. The finite-size scaling law is adopted to determine $T_{\mathrm{c}}$ and the crossover exponent, $\phi$. We obtain $T_{\mathrm{c}}=1.625$ and $\phi=0.51$ for the infinitely long copolymer $\mathrm{A}_{n} \mathrm{~B}_{n}$. Our results show that the critical adsorption temperature and exponents of the diblock copolymer $\mathrm{A}_{n} \mathrm{~B}_{n}$ with a large $n$ value are determined by the A block that has a stronger attraction. To investigate the effect of the $\mathrm{B}$ block on the finitely long copolymers, we compared the contact number of $\mathrm{A}_{n} \mathrm{~B}_{n}$ with that of homogeneous polymer $\mathrm{A}_{n}$. We found that the non-adsorbed B block weakens the adsorption of the A block; however, the effective influence of the $\mathrm{B}$ block on $\left\langle M_{\mathrm{A}}\right\rangle$ is independent of $n$ at $T_{\mathcal{c}}$, resulting in the same $T_{\mathrm{c}}$ and $\phi$ for both $\mathrm{A}_{n} \mathrm{~B}_{n}$ and $\mathrm{A}_{n}$ at large $n$. Simulations on the general copolymer $\mathrm{A}_{n} \mathrm{~B}_{m}$ give the same conclusion that the non-adsorbed $B$ block does not influence the $T_{\mathrm{c}}$ of a long $\mathrm{A}_{n} \mathrm{~B}_{m}$.

The non-adsorbed $\mathrm{B}$ block also influences the conformational properties of the end-grafted copolymer $\mathrm{A}_{n} \mathrm{~B}_{m}$. At high temperature, the $\mathrm{B}$ block increases the size of the $\mathrm{A}$ block and the asphericity parameter. At low temperatures, the B block prevents the adsorption of tail monomers in the A block; thus, the size and the asphericity parameters decrease with the length of the B block. At CAP, the asphericity parameter $\langle\mathrm{A}\rangle$ is independent of the $\mathrm{B}$ block, which is in agreement with the properties of homogeneous polymer. The temperature variation of size and shape shows a phase transition of the A block from a $3 \mathrm{D}$ configuration at high temperatures to a $2 \mathrm{D}$ configuration at low temperatures. However, the influence of block $\mathrm{B}$ dies away with an increase in the length of the A block.

\section{ACKNOWLEDGEMENTS}

This work was supported by the National Natural Science Foundation of China under grant nos 11304231, 21171145 and 11374255 and by the Zhejiang Natural Science Foundation of China under grant no. LY14B040004.
1 Lehner, R., Koota, J., Maret, G., Gisler, T. Segment distributions of end-tethered polymers in a good solvent. Phys. Lett. Rev. 96, 107801 (2006).

2 Pasquardini, L., Lunelli, L., Potrich, C., Marocchi, L., Fiorilli, S., Vozzi, D., Vanzetti, L., Gasparini, P., Anderle, M., Pederzolli, C. Organo-silane coated substrates for DNA purificatio. Appl. Surf. Sci. 257, 10821-10827 (2011).

3 Sevastianov, V. I. Biocompatible biomaterials: current status and future perspectives. Trend Biomater. Artif. Organs. 15, 20-30 (2002).

$4 \mathrm{Hsu}, \mathrm{H}$. P., Binder, K. Effect of chain stiffness on the adsorption transition of polymer. Macromolecules 46, 2496-2515 (2013).

5 Pasch, H. Hyphenated techniques in liquid chromatography of polymers. Adv. Polym. Sci. 150, 1-66 (2000).

6 Liu, W., Zhao, H., Yang, Y., Liu, X., Xu, B. Reactive carbon microspheres prepared by surface-grafting 4-(chloromethyl)phenyltrimethoxysilane for preparing molecularly imprinted polymer. Appl. Surf. Sci. 277, 146-154 (2013).

7 Galloway, J. A., Jeon, H. K., Bell, J. R., Macosko, C.W. Block copolymer compatibilization of cocontinuous polymer blends. Polymer 46, 183-191 (2005).

$8 \mathrm{Pu}, \mathrm{G}$., Luo, Y., Wang, A., Li, B. Tuning polymer blends to cocontinuous morphology by asymmetric diblock copolymers as the surfactants. Macromolecules 44, 2934-2943 (2011)

9 Ruiz-Taylor, L. A., Martin, T. L., Zaugg, F. G., Witte, K., Indermuhle, P., Nock, S., Wagner, P. Monolayers of derivatized poly(L-lysine)-grafted poly(ethylene glycol) on metal oxides as a class of biomolecular interfaces. Proc. Natl Acad. Sci. USA 98 852 (2001).

10 Klushin, L. I., Polotsky, A. A., Hsu, H. P., Markelov, D. A., Binder, K., Skvortsov, A. M. Adsorption of a single polymer chain on a surface: Effects of the potential range. Phys. Rev. E 87, 022604 (2013).

11 Eisenriegler, E., Kremer, K., Binder, K. Adsorption of polymer chains at surfaces: scaling and Monte Carlo analyses. J. Chem. Phys. 77, 6296-6320 (1982).

12 Möddel, M., Janke, W., Bachmann, M. Adsorption of finite polymers in different thermodynamic ensembles. Comput. Phys. Commun. 182, 1961-1965 (2011).

13 Milchev, A., Binder, K. Static and dynamic properties of adsorbed chains at surfaces Monte Carlo simulation of a Bead-Spring model. Macromolecules 29, 343 (1996).

14 Decase, R., Sommer, J. U., Blumen, A. Static and dynamic properties of tethered chains at adsorbing surfaces: a Monte Carlo study. J. Chem. Phys. 120, 8831 (2004).

15 Decase, R., Sommer, J. U., Blumen, A., Grafted polymer chains interacting with substrates: computer simulations and scaling, Macromol. Theory Simul. 17, 429 (2008).

16 Seaton, D. T., Wust, T., Landau, D. P. A Wang-Landau study of the phase transition in a flexibale homopolymer. Comput. Phys. Commun. 180, 587 (2009).

17 Li, H., Qian, C. J., Sun, L. Z., Luo, M. B. Simulation of a flexible polymer tethered to a flat adsorbing surface. J. Appl. Polym. Sci. 124, 282 (2012).

18 Luo, M. B. The critical adsorption point of self-avoiding walks: a finite-size scaling approach. J. Chem. Phys. 128, 044912 (2008).

$19 \mathrm{Li}$, H., Qian, C. J., Luo, M. B. Conformational properties of a polymer tethered to an interacting flat surface. Polymer J. 42, 383 (2010).

20 Gong, Y. C., Wang, Y. M. Partitioning of polymers into pores near the critical adsorption point. Macromolecules 35, 492 (2002).

21 van Rensburg, E. J. J., Rechnitzer, A. R. Multiple Markov chain Monte Carlo study of adsorbing self-avoiding walks in two and in three dimensions. J. Phys. A: Math. Gen. 37, 875 (2004)

22 Metzger, S., Müller, M., Binder, K., Baschnagel, J. Adsorption transition of a polyme chain at a weakly attractive surface: Monte Carlo simulation of off-Lattice models. Macromol. Theory Simul. 11, 985 (2002).

23 Evers, O. A., Scheutjens, J. M. H. M., Fleer, G. J. Statistical thermodynamics of block copolymer adsorption. 1. Formulation of the model and results for the adsorbed layer structure. Macromolecules 23, 5221-5233 (1990).

24 Bhattacharya, S., Hsu, H.-P., Milchev, A., Rostiashvili, V. G., Vilgis, T. A. Adsorption of multiblock and random copolymer on a solid surface: critical behavior and phase diagram. Macromolecules 41, 2920-2930 (2008).

25 Sumithra, K., Baumgaertner, A. Adsorption of random copolymers: A scaling analysis. J. Chem. Phys. 110, 2727-2731 (1999).

26 Milchev, A., Rostiashvili, V., Bhattacharya, S., Vilgis, T. Polymer chain adsorption on a solid surface: scaling arguments and computer simulations, nanophenomena at surface. Springer Ser. Surf. Sci. 47, 185-204 (2011).

27 Sumithra, K., Straube, E. Adsorption of diblock copolymers on stripe-patterned surfaces. J. Chem. Phys. 125, 154701 (2006).

28 Shaffer, J. S. Effects of chain topology on polymer dynamics: bulk melts. J. Chem. Phys. 101, 4205-4213 (1994).

29 Rosenbluth, M. N., Rosenbluth, A. W. Monte Carlo calculation of the average extension of molecular chains. J. Chem. Phys. 23, 356-359 (1954).

$30 \mathrm{Li}, \mathrm{H}$., Qian1, C.J., Wang, C., Luo, M. B. Critical adsorption of a flexible polymer confined between two parallel interacting surfaces. Phys. Rev. E 87, 012602 (2013).

31 Bishop, M., Saltiel, C. J. Polymer shapes in two, four, and five dimensions. J. Chem Phys. 88, 3976 (1988).

32 Huang, J. H., Luo, M. B., Wang, Y. M. Dissipative particle dynamics simulation on a ternary system with nanoparticles, double-hydrophilic block copolymers, and solvent. J. Phys. Chem. B 112, 6735-6741 (2008). 